\title{
La investigación urbana y el poder local: un acercamiento desde la sociología del desarrollo
}

\section{Introducción}

La discusión acerca del fenómeno del poder local ha producido recientemente nuevas reflexiones acerca de sus alcances y limitaciones. Estimulada por las iniciativas que municipios y amplios sectores ciudadanos han impulsado, el tema ha adquirido una renovada dimensión; sobre todo porque constituye uno de los pilares básicos de los procesos de la reforma social que se desarrollan en América Latina. En países como El Salvador, por ejemplo, políticos, analistas, empresarios y sectores de la sociedad civil se encuentran, por diversos motivos, en un escenario de conflicto respecto a la problemática municipal y local. En este caso, el desarrollo de dicho conflicto y sus eventuales salidas será un factor clave para el avance, estancamiento o retroceso de la transición democrática.

En la actualidad, la sociología del desarrollo ha incorporado dentro de sus reflexiones los aspectos urbanos y de poder local debido a su importancia en la configuración de los modelos altemativos de desanollo. Lo importante desde esta perspectiva no sólo es producir trabajos académicos, sino abrir espacios institucionales; es decir, un compromiso social para que el trabajo académico sea una contribución a la comprensión y la resolución de aquellos problemas en lo que la especificidad de lo urbano-regional sea considerado a partir de una preocupación más global: lograr una distribución más justa de la riqueza en el contexto de una sociedad democrática.

Tal como ha sido planteado por varios analistas, los paises de la periferia tienen dos problemas básicos que resolver en su agenda de desarrollo: en primer lugar, la construcción de una democracia activa y en segundo lugar, la adopción de un estilo de desarrollo económico que beneficie a los grandes sectores de la población y resuelva los endémicos problemas estructurales. El fortalecimiento del poder local y consecuentemente el papel de los actores- sujetos para lograr dicho 
propósito, vendría a contribuir, en gran medida, a resolver los problemas básicos planteados.

El propósito de este trabajo es presentar una serie de reflexiones generales, desde la perspectiva de la sociología del desarrollo, acerca de la orientación y las perspectivas que prevalecen en tomo a la temática del poder local en el marco de la investigación urbana, tomando como referencia el contexto latinoamericano, pero pensando en los procesos que se desarrollan en El Salvador. El interés que se persigue con estas notas es organizar un conjunto de ideas que puedan ser discutidas colectivamente con el propósito de orientar el rumbo de la investigación por realizar en el futuro. Asimismo, el carácter reflexivo del presente artículo explica el escaso espacio a las referencias bibliográficas. Dada la abundante literatura existente, y mi propósito de evitar la pesadez académica, he optado por limitar las referencias al estricto mínimo.

\section{La problemática del poder local}

El tema del poder local en las comunidades fue tratado en varios trabajos de la sociología clásica y funcionalista. Sin embargo, esta tradición metodológica en las investigaciones urbanas ha tenido muy poca influencia en las investigaciones urbanas realizadas fuera del contexto norteamericano. En el caso latinoamericano, la principal influencia la ha tenido la escuela francesa.

Los trabajos del joven Manuel Castells marcaron el inicio de investigaciones que, tomando como contexto de análisis la ciudad, reinterpretaban desde el marxismo los procesos económicos y sociales del espacio urbano. Las concepciones totalizadoras de la realidad adoptadas en la época prevalecieron también en el análisis del espacio urbano. Los agentes y el comportamiento de los mismos fue analizado desde proposiciones teóricas de elevado nivel de abstracción. El esfuerzo empírico consistía, en todo caso, en comprobar que el análisis de las relaciones sociales existentes en el espacio urbano corrrespondía a leyes generales de la acumulación capitalista. Pero algo no poco trascendente enmarcó aquella producción: la cuestión urbana pasó a ser una cuestión política (Bettin, 1982). El poder apareció como una categoría fundamental en el estado capitalista. La política urbana del mismo constituía una forma de intervención en la organización y el uso del suelo urbano en función de las exigencias de las clases dominantes. Sólo el poder de los movimientos sociales urbanos podía modificar esta situación y, por ello, eran verdaderos agentes sociales de cambio. Hasta finales de los setenta pocos son los trabajos que se interesan por estudiar la incidencia que ejercen diferentes actores sociales sobre la estructuración y el uso de un espacio local (urbano). También son pocos aquellos que el estudio de la política urbana se instalan en la actuación específica y, muchas veces, contradictoria de las instituciones gubernamentales. 
Desde hace aproximadamente una década, el poder en relación a la investigación urbana gana un nuevo contenido. El poder local, visto genéricamente como la capacidad de manejar recursos en función de un interés dado, lleva al estudio de órganos e instituciones locales encargados de la gestión de su territorio. Las políticas de descentralización económica y social, que comenzaron a estructurarse como salida de la crisis, demandaban una propuesta y ella se ubicó necesariamente en la transferencia de recursos y responsabilidades a las instancias menores (departamentos, municipios, etcétera). Ahora es la condición de la ciudadanía social la que importa analizar. Esta es una identidad previa a la consitución de cualquier movimiento o agrupamiento social. La trascendencia del tema está asociada nuevamente a la influencia intelectual de autores europeos que continúan estableciendo como objeto de estudio el devenir de América Latina. Jordi Borda no sólo sembró nuevas ideas sobre la importancia de abordar el tema, sino que promovió la realización de una investigación comparativa en estos contextos nacionales $y$, en la actualidad, ha contribuido a actualizar sus ideas incorporando nuevos elementos (Borda y Castells, 1997). Pero, ¿cuál es la situación que prevalece en nuestros países? ¿Cuál es la coyuntura que hace que el tema gane importancia? En términos muy generales: el binomio neoliberalismo económico/democratización política. La adopción del modelo neoliberal globalizado que presentan la mayoría de los países latinoamericanos exige una reestructuración profunda de nuestras economías y nuestras sociedades. El patrón capitalista de acumulación adoptado en los países periféricos como altemativa al viejo modelo muestran inequivocos signos de agotamiento; los alcances de la reforma económica impulsada en la década de los noventa muestra cada vez más sus límites inmanentes. Por otra parte, la presente situación internacional agrega crecientes cargas y dificultades a nuestras economías y márgenes de actuación cada vez más limitados.

Desde una perspectiva espacial, la concentración del poder económico y político en un espacio territorial dado es cuestionada. Una ciudad o unas pocas, generan y absorven la mayor parte de los recursos del país. Mientras tanto, son pocos los espacios interiores con una estructura productiva y social más o menos equilibrada. Estas son las características más relevantes de este patrón de urbanización capitalista periférico. Precisamente comprender el carácter "deformado"de nuestra realidad espacial obliga a retomar varias de las contribuciones de la teoría del desarrollo e incorporarlas a los esfuerzos que se realizan en el área de los estudios urbanos.

Urbanistas, técnicos y principalmente políticos ven hoy la concentración como una forma de ocupación territorial, que actúa de manera negativa al exigir permanentes y elevadas inversiones para mantener a la ciudad o las ciudades claves. En estos espacios se concentran intensos conflictos sociales tras la demanda creciente de bienes y servicios: también en los mismos el elevado número de la población provoca efectos multiplicadores ante cualquier fenómeno de tipo natu- 
ral. Por ello, las políticas de descentralización son incorporadas en la agenda gubernamental de distintos regímenes políticos, ya sea por la necesidad de responder ante problemas internos o porque existe presión por parte de los organismos financieros multilaterales.

La descentralización, entendida aquí en sentido amplio y en sus diferentes niveles como desconcentración de actividades, población y transferencia de recursos de poder, exige elaborar propuestas territoriales alternativas (Perló y Ziccardi, 1987). Los investigadores de la cuestión urbana ya han comenzado a dedicar mayor atención a las ciudades pequeñas. Pero son los estudios regionales los que proveen un conocimiento preliminar e indispensable para la elaboración de cualquier política descentralizadora.

Las crisis e incertidumbres presentes señalan los límites posibles de aquellas políticas públicas, dirigidas a promover la desconcentración de actividades y población. La escasez de recursos, el elevado costo que posee la producción de nuevas infraestructuras productivas y sociales constituyen los principales obstáculos para cualquier proyecto desconcentrador: Por otra parte, no es evidente aún que nuestras grandes ciudades generen deseconomías, que sean disfuncionales, que provoquen disminuciones en los niveles de rentabilidad del capital localizado en las mismas: todo indica lo contrario, son el espacio adecuado para lograr los objetivos de la producción capitalista.

Ahora bien, la experiencia en la región nos indica que la descentralización, en su acepción restringida de desconcentración económica, está directamente asociada a la problemática económica y/o a los imperativos de la nueva racionalidad económica que impone a nuestras sociedades el contexto internacional. Las exigencias de la reconversión industrial o de la modemización tecnológica nacional no son los fundamentos de las políticas públicas. En la actualidad, éstas son una expresión de la lógica del modelo prevaleciente y, en tal sentido, son funcionales a la readecuación de los procesos de acumulación de capital.

Una política de descentralización debe considerar necesariamente la cuestión regional y local. Sin embargo, las políticas públicas son sectoriales y en su interior la dimensión territorial no siempre es incorporada con la importancia que la misma merece. Por otra parte, la descentralización económica suele limitarse a modificar la asignación territorial de los recursos fiscales de la nación, de manera que el gobierno central ejerza una acción más equitativa entre sus entidades; pero ello no significa modificaciones sustanciales en la estructura productiva de las diferentes regiones del país.

La descentralización en la región adquiere un interés sustancial cuando se vincula a los procesos de democratización política. La descentralización es, en sí, transferencia de poder de las entidades centrales a los gobiernos locales. Es delegación de funciones, asignación justa de recursos, autonomía de los entes 
locales para organizar las actividades y los servicios de sus localidades. Es más bien en este sentido en el que la investigación urbana debe enfocar el tema del poder local.

La centralización, que fue una condición necesaria en los albores de la formación de los Estados nacionales, ahora es considerada como una cuestión que contribuye poco a la profundización y expansión de los procesos democráticos. El tema de la democracia obliga a repensar el papel del gobierno central y de las clases dominantes, cuyo poder económico y político se concentró en determinados espacios del territorio nacional. La ciudadanía, para acercarse a sus gobernantes, demandar sus derechos y participar en los procesos decisorios, debe acceder directamente a las instancias gubemamentales más próximas. Ello implica revisar prácticas burocráticas de gestión pública y hacer del ejercicio de los derechos ciudadanos una posibilidad de incidir, de manera directa, sobre el proceso de toma de decisiones del territorio que habitan.

Para los investigadores urbanos, la temática del poder local abre un nuevo escenario y nuevas interrogantes para el análisis. Los estudios que habían predominado en nuestros países seguían dos tipos de línea de investigación: (1) Los que tomaban las grandes ciudades latinoamericanas, estudiaban los componentes de su estructura urbana y el comportamiento de diferentes actores sociales, particularmente la vivienda popular a través del estudio de las políticas del gobierno, sus planes y programas, el monto de las inversiones, el número de acciones, las necesidades habitacionales y las organizaciones sociales de los pobladores. También, en menor medida, el transporte y los servicios han sido temas analizados profundamente. En esta línea de investigación la cuestión del poder local y el municipio como unidad de análisis no es abordado. (2) Los estudios regionales que se acercan más a la problemática del poder en tanto los enmarcan. Sin embargo, no se ha establecido una articulación entre esta producción y la dedicada específicamente al poder local.

El poder local ha sido analizado a partir de una reflexión que considera principalmente los municipios urbanos, con todas las limitaciones que implica. Talvez puede decirse que tradicionalmente la investigación urbana se vincula mucho más a las interrogantes que surgen de la teoría y la práctica política, mientras que la investigación regional encuentra sus fundamentos teóricos metodológicos en la economía clásica y la economía política.

\section{La dimensión municipal: una evaluación preliminar}

El tema del poder local ha trasladado la atención de la investigación urbana actual al territorio municipal. Pero debe recordarse que en América Latina han existido estudiosos de la cuestión municipal desde mucho tiempo atrás. Diogo Lordello de Mello, en Brasil, o Gustavo Martínez, en México, son nombres asociados a este importante pensamiento municipalista. El estudio de los proble- 
mas municipales surge vinculado a las preocupaciones de los administradores públicos, interesados en conocer la actuación gubernamental en sus distintos niveles e instituciones. De igual forma, los juristas han realizado profundos estudios del marco legal que rige el funcionamiento de la estructura del gobierno. Sus contribuciones se han traducido en modificaciones sustanciales respecto a la legislación municipal. En el marco de estas dos disciplinas, administradores públicos y juristas han desarrollado trabajos tendientes a adecuar las diferentes instancias gubernamentales a las modalidades que adquirió el desarrollo económico y el sistema político-social en nuestros países.

Para los urbanistas, este tema ha adquirido interés recientemente. La abundante bibliografía sobre desarrollo regional ofrece un marco de referencia global para el análisis de los espacios interiores menores que son los municipios (Hardoy, 1986). Esta producción académica anterior ha ofrecido elementos para los primeros diagnósticos sobre poder local. Muchas ideas, que los trabajos recientes profundizan, habían sido ya esbozadas o desarrolladas desde hace por lo menos una década. Lo difícil de nuestra tarea será reinterpretarlas desde las preocupaciones que hoy orientan nuestras investigaciones: la búsqueda de opciones económicas viables y la democratización en nuestros respectivos países y, desde este marco general, explorar la dimensión urbano-regional.

Una primera idea presente en las preocupaciones actuales de los gobiernos locales, es que urge una modernización de la estructura de gobierno de este nivel. La idea central sostenida por Lordello de Mello (1983), en un importante trabajo dedicado a analizar el tema, es que modernizar los gobiernos locales en América Latina no es un problema de métodos y procesos, de organizacción técnica y/o administrativa, sino que hace referencia a criterios de eficacia y eficiencia en la prestación de servicios públicos y de equidad y justicia en la distribución de los mismos. El autor señala que lo que debe modificarse son los procesos de toma de decisiones, los cuales deben ser repensados a la luz de las exigencias de participación ciudadana. En este sentido, Lordello de Mello supera el análisis funcional de la administración pública y se instala en las modalidades que adquieren los procesos de gestión municipal históricamente considerados. Dos cuestiones señaladas por él son de fundamental importancia para incorporarlas en la investigación urbana: (1) Los señalamientos que efectúa sobre los imperativos de la modernización, entre ellos, la urbanización, el incremento de la población en las ciudades, la complejidad de las actividades gubernamentales, la ampliación de la demanda de los servicios públicos y la elevación de los niveles en su prestación, y la participación ciudadana en el gobierno local más allá de lo electoral (estas cuestiones son consideradas imperativos válidos para promover la modernización de los poderes locales) y (2) los llamados imperativos estructurales, una serie de síndromes que comparten la mayoría de los municipios latinoamericanos, tales como poder formal municipal y centralismo gubernamental real, anacronismo en la estructura básica del gobierno local, fracciona- 
miento del poder y falta de coordinación, inestabilidad administrativa de los funcionarios y de las esferas superiores de decisión, simetría o tratamiento uniforme a realidades heterogéneas, planificación nacional sin referente territorial, desmunicipalización de los servicios públicos, abdicación del municipio en el ejercicio de sus funciones que le competen por carecer de recursos financieros y humanos, dependencia y control del gobierno central en su gestión.

En general, la experiencia ha demostrado que los municipios existen jurídica y administrativamente como parte constitutiva del gobiemo nacional. El concentrado desarrollo económico se acompañó de un proceso de centralización política en que estructuras político-administrativas menores, como el municipio, sólo cumplen funciones asignadas formalmente con muchas dificultades. Las excepciones son los grandes y poderosos municipios, las capitales nacionales o los territorios estratégicos para alguna actividad económica y/o políticamente específica.

Uno de los temas que más ha preocupado a los especialistas en el área, es la relación gobierno central/gobierno municipal. Hace algunos años esta temática fue discutida ampliamente por los investigadores de la región y de fuera, por políticos y funcionarios vinculados directamente a la gestión municipal (CERLAC, 1996). Según sus conclusiones: (1) la dimensión política de la relación presentaba una marcada separación entre los intereses de los representantes y los de la propia comunidad que los eligió a través del voto; (2) en lo económico, la vida municipal presentaba una marcada escasez de recursos, las participaciones en los ingresos públicos nacionales eran muy bajas; existía incapacidad para generar recursos propios por limitaciones geográficas o de recursos y, (3) en lo administrativo se señalaba precisamente la insuficiente capacidad técnico-administrativa, el excesivo peso del gasto corriente, la falta de remuneraciones adecuadas a las autoridades locales, la concentración de poder en el ejecutivo local.

Todos estos trabajos ofrecieron cierta acumulación de conocimiento sobre las características que compartían las entidades municipales de los países latinoamericanos. Sin embargo, ello no era suficiente para ofrecer altemativas tendientes al fortalecimiento del poder local, capaces de ser incorporadas a los proyectos gubernamentales democráticos y reivindicadas por las fuerzas sociales progresistas. Era necesario realizar un esfuerzo de análisis desde mayores niveles de especificidad, en cada realidad nacional.

En los trabajos del evento mencionado, se planteó que el modelo económico constituye el marco de referencia para cualquier estudio que intente avanzar en esta problemática. Así, para los gobiemos locales ello se traduce en una mayor escasez de recursos, en una débil capacidad de gestión y en una escasa eficacia social. Los gobiemos locales son simples receptores pasivos de decisiones políticas que se procesan en niveles superiores de gobierno y eventuales destinatarios de los fondos que aquéllas les asignan para su propia gestión; permanecen 
inhabilitados para poder dar respuesta satisfactoria al conjunto de demandas sociales de la población. Pero, en contrapartida, están en contacto directo con dichas necesidades y demandadas, por cuanto constituyen los niveles territoriales inferiores de la administración pública y, por lo tanto, los más próximos a los ámbitos específicos del espacio social donde se verifican las condiciones concretas de existencia de la población.

Dos líneas de análisis parecen ir ganando aceptación entre los especialistas: (1) retomar, en cada caso concreto, los problemas relacionados con la modernización técnica de la gestión de los gobiemos locales de la región (estudio de instrumentos jurídicos, organización administrativa, funciones de gestión pública, etcétera); y (2) considerar con especial atención la problemática de la cultura política, en el sentido de analizar los procesos sociales y políticos dentro de la perspectiva de ejercer una democracia participativa en la gestión local, reasignar competencias, revisar las relaciones entre niveles, garantizar la participación de la comunidad en la toma de decisiones, promover relaciones interpartidarias en el municipio, etcétera.

Lo fundamental, desde la perspectiva de la investigación urbana, era la reinterpretación de la compleja problemática de la democracia local, otorgando particular atención a la dimensión territorial. El esfuerzo interdisciplinario deberá agregar a los análisis de administradores públicos, juristas y politólogos, el de los urbanistas.

\section{Avances y perspectivas}

Esta sección pretende recoger sólo algunos de los temas cuyo tratamiento puede ser revisado a partir de la confrontación con las complejidades de las realidades concretas. Las cuestiones que se quieren abordar son exclusivamente referidas a la dimensión territorial que encierra el tema del poder local, principalmente desde las preocupaciones de la investigación urbana y de la sociología del desarrollo. Se trata de: (1) aproximarse a las experiencias, a fin de revisar algunas proposiciones comúnmente aceptadas sobre el problema del poder local, y (2) considerar las perspectivas de investigación urbana en relación con este objeto de estudio.

\section{I}

(a) La debilidad del poder local del municipio, argumento principal de nuestra reflexión, debe ser calificada. El análisis indica que existen municipios fuertes, en términos de los recursos de que disponen y con el relativo poder político que sustentan. Las capitales nacionales son ejemplo de ello. Por supuesto que esto tiene su origen en condiciones históricas concretas y en las modalidades en las cuales los espacios nacionales se han articulado al sistema capitalista mundial. 
En algunos casos, esta situación se extiende incluso en los municipios periféricos de la gran ciudad. Esto es de fundamental importancia si se piensa en la relación poder local/urbanización.

Como ha sido tradicional, las investigaciones han privilegiado el análisis de las áreas metropolitanas. Sin embargo, los aspectos político-administrativos y la naturaleza particular de los gobiernos han sido poco estudiados. La gran ciudad es observada en tanto territorio del gobierno central. Esta se ha estudiado más en su unidad que en su fragmentación. Aquí hay, entonces, un vacío que debemos considerar en el futuro.

Si la investigación amplía su marco de referencia e intenta introducir la variable territorial, el análisis del poder local cuenta con una realidad mucho más compleja. La fuerte presencia que tienen los municipios rurales en los países de la región también debe ser abordada con toda la importancia que encierra la misma. Estos son precisamente los municipios débiles, aquellos en que los gobiernos locales tienen muy poco para administrar y donde la sobrevivencia depende de los recursos que le transfiere el gobierno central. Pero estos municipios son los que prácticamente no han sido analizados porque existe poca información disponible y porque se ha asumido que su peso económico, político y cultural ha sido marginal. Solo la investigación regional ha dado los primeros pasos en analizar estos territorios nacionales. Por otra parte, entre uno y otro extremo está la situación de los municipios que albergan situaciones intermedias, aun cuando se les pueda considerar urbanos. Su análisis no necesariamente con el de las llamadas ciudades medias, pero en estos espacios urbanos, la debilidad del gobierno local y/o del poder local no es una situación generalizada.

En síntesis, si se analiza la relación poder local/urbanización se deben estudiar los municipios fuertes, los que tienen una situación relativamente importante dentro de la organización político-administrativa y económica del territorio nacional. Pero, al mismo tiempo, el tema del poder local debe exceder al de los espacios urbanos, razón por la cual la investigación urbana deberá recuperar los estudios regionales e incorporar a los mismos la fragmentación territorial municipal.

Metodológicamente este planteamiento nos lleva a recuperar a las tipologías con todas las limitaciones que este instrumento de análisis presenta. Pero en el campo de la investigación, no se pueden repetir aquellos errores que Diogo Lordello de Mello calificaría de "simetría" para referirse al igual tratamiento que reciben entidades político-administrativas heterogéneas. Algunos autores iniciaron, hace algunos años, un ejercicio de este tipo (Martínez Assad y Ziccardi, 1987) y en años recientes han incorporado algunas contribuciones importantes (Ziccardi, 1995). Sin embargo, es importante tal vez abrir una discusión de tipo metodológico para elaborar criterios que contribuyan a crear agrupamientos territoriales municipales con cierto grado de homogeneidad, a partir de la incorporación de variables económicas, políticas, sociales y culturales. 
(b) Partimos de la dependencia del gobierno local respecto al central, de su ineficiencia burocrática y de su incapacidad económica. Pero, en contrapartida, el municipio es considerado como la instancia del gobierno próxima a la ciudadanía y, por lo tanto, accesible a sus demandas. Esto permite que potencialmente pueda inaugurarse un ejercicio de prácticas democráticas en la gestión del territorio. Estas proposiciones generales deben ser revisadas en la investigación urbana. La concentración y atención de los llamados "medios de consumo colectivo" han constituido un núcleo de análisis relevante en varias investigaciones recientes. Se puede pensar, entonces, en los procesos descritos a partir de estos componentes de la gestión urbana y dejar a los politólogos la ardua tarea de recomponer el concepto de democracia a partir de todas las dimensiones, hechos y comportamientos sociales que el mismo encierra.

Mucho del marco de referencia actual debe ser sometido a un sistemático análisis para su aprobación o rechazo. Las investigaciones realizadas no permiten aún emprender este trabajo, puesto que es una cuestión que demanda mayor profundidad en la investigación empírica, principalmente de la sistematización de la información local a partir de investigaciones de carácter cualitativo. Lo cierto es que impulsar la desconcentración de los servicios (salud, educación y, en algunos casos, la acción pública en materia habitacional) es una idea aceptada consensualmente para la elaboración y realización de políticas públicas. Se comienza por desconcentrar las actividades gubernamentales de tipo administrativo y la provisión y suministro de los medios de consumo colectivo. Desde el enfoque de la sociología del desarrollo, en este proceso se puede evaluar la eficiencia administrativa y la eficacia social, la disponibilidad real de recursos financieros y también el vínculo gobierno local/ciudadanía.

El tema de la provisión de los bienes de consumo colectivo encierra la participación de un conjunto de instituciones gubernamentales de diferente nivel. Su presencia en el espacio municipal demanda, como lo han señalado varios autores, que exista coordinación institucional para lo cual se requiere que la autoridad local posea cierto grado de reconocimiento en el interior de la burocracia gubernamental. No solamente estará en juego el comportamiento administrativo, sino también político. La ciudadanía demanda bienes y servicios y no necesariamente mayor autonomía de su gobiemo local ni mayor participación. Aun en países con una fuerte tradición en movilización popular y movimientos sociales urbanos, la descentralización no tiene peso político como demanda de la sociedad civil y mucho menos entre los sectores populares. Los temas que movilizan la mayor autonomía y la reforma fiscal son demandas de las propias autoridades locales: los alcaldes. Pero el tema de los bienes de consumo y el poder local remite al de las obras públicas, a su realización, a la participación de diferentes actores económicos y sociales en su decisión y concreción, a quienes se benefician, cuál es la distribución espacial y social de la inversión pública: tema prácticamente desconocido en la investigación urbana y que estudiado a nivel local 
facilitaría su comprensión. Los medios de consumo colectivo han interesado más a los ensayos teóricos que a la investigación empírica. En este último tipo de análisis lo que ha importado son las experiencias de organización y lucha de las clases populares y las modalidades de actuación gubernamental, más desde un punto de vista institucional que desde la distribución territorial de los bienes. Por ello, se debe vincular la cuestión del poder al suministro de bienes y servicios, considerando no sólo la distribución y consumo, sino su propia producción.

II

En relación con las perspectivas futuras de la investigación urbana desde la perspectiva de la sociología del desarrollo sobre el tema del poder local, muchas son las interrogantes que deben responderse para pasar de un nivel analíticodescriptivo a uno explicativo.

En principio, existe un conjunto de cuestiones fundamentales sobre los niveles intermedios de análisis: departamental o provincial. En general, existe cierta tendencia a afirmar que el fortalecimiento del poder local municipal se traduce en un debilitamiento del Poder Legislativo o inclusive del propio Ejecutivo local. El papel de las instancias intermedias, sus relaciones con los municipios es poco abordado. La autoridad provincial, departamental o estatal suele tener reservas respecto al fortalecimiento de los municipios.

Esta reflexión nos ubica en un tema que los urbanistas, al analizar la dimensión espacial del problema del poder local, han desarrollado poco y la sociología del desarrollo considera crucial: las características de la estructura económica local. Llama la atención la poca importancia otorgada por los especialistas al estudio de los procesos sobresalientes de la economía local. La población del municipio es considerada desde una perspectiva demográfica cuantitativa, pero no existen análisis en términos de las características que posee la fuerza de trabajo, las modalidades que presenta el mercado de trabajo local, los niveles salariales y las condiciones de vida que prevalecen, el comportamiento de las formas de organización y la influencia de los componentes culturales. Entonces, de lo que se trata es de agregar el comportamiento de la economía local a la temática de la modernización administrativa del aparato gubernamental local y de la cultura política.

Una segunda idea es que la descentralización parece estar inserta en un "clima ideológico favorable", pero tropieza sistemáticamente con los obstáculos que le imponen a su concreción la propia dinámica burocrática, la escasez de recursos y las limitaciones inherentes del modelo económico imperante. Se ha planteado que el tema de los bienes de consumo colectivo, desde la perspectiva de la investigación urbana, puede hallar en el municipio un escenario de análisis. En relación al acceso, distribución, obtención de estos bienes de consumo colectivo se instalan una serie de prácticas políticas clientelistas o paternalistas, 
que han llegado inclusive a atribuirle al municipio el papel de amortiguador de los conflictos sociales, lo cual - vale la pena señalarlo- está presente en las concepciones neoliberales que abordan el problema de la gobernabilidad (Carrión, 1985; Alfaro, 1995).

Finalmente, existe cierta creencia de que la representación partidaria parlamentaria está en crisis. Todo ello obliga a orientar el análisis de las políticas de descentralización no sólo sectorialmente (salud, educación, vivienda), sino a esforzarnos a poner a prueba su viabilidad en determinados espacios geográficos. El municipio puede ser un contexto en los procesos de concertación nacional en el cual se incorporen otros actores distintos a los fundamentales (capital/trabajo) y puede ser el espacio donde la descentralización promueva procesos de concertación que permitan satisfacer las demandas ciudadanas y promover procesos de fortalecimiento democrático (Dos Santos, 1987).

Resumiendo, la situación actual obliga a reconocer que existe un largo camino por recorrer en los estudios sobre las cuestiones urbanas y el poder local. Sin embargo, el esfuerzo deberá orientarse a formular un discurso que sea capaz de incorporar los esfuerzos colectivos provenientes de las diferentes disciplinas de la teoría social y de la praxis de los movimientos y agnupaciones sociales. Por su parte, la sociología del desarrollo intenta enriquecer su análisis mediante la introducción de problemáticas que contribuyan a configurar un paradigma emergente, que sirva como instrumento de cambio para nuestras sociedades.

\section{Nota}

1. Lecturer del Departamento de Sociologia y Estudios Sociales, Universidad de Regina (Regina, Canadá) y profesor de la Maestria en Gestión del Medio Ambiente de la Universidad Centroamericana "José Simeón Cañas" (UCA).

\section{Referencias bibliográficas}

Alfaro, S. O., Development and Democracy in the Third World. Documento presentado al Seminario Permanente del Programa de Doctorado en Sociología. York University, Toronto, Canadá, 1995.

Bettin, G. Revisiones teóricas y resultados empíricos en sociologia. UACH, 1982 (mimeo).

Borja, J. "Transformaciones terrritoriales e instituciones en Europa". En Revista Mexicana de Sociología, Año 48, Vol. 4, 1986, pp. 225-257.

Borja, J. y Castells, M. Local y global. La gestión de las ciudades en la era de la información. Taurus, Madrid, 1997.

Carrión, F. "Alcances de la problemática municipal en las ciudades ecuatorianas". En Documentos (s/l), Centro de Investigaciones, No. 14, 1985.

Centro de Investigaciones para América Latina y el Caribe (CERLAC). York University, Seminario para el Desarrollo de la Democracia Local, Toronto, 1996.

Dos Santos, F. Concertación político-social y democratización. CLACSO, Buenos Aires, 1987. 
Hardoy, J. "La Comisión de Desarrollo Urbano y Regional: 18 años de experiencia". En David y Goliath, 49, julio, Buenos Aires, 1986, pp. 63-68.

Lordello de Mello, D. "Modernización de los gobiernos locales en América Latina". En Revista SLAP, 66, Vol 17, 1983, pp. 185-202.

Martínez Assad, C. y Ziccardi, A. "El municipio entre la sociedad y el Estado". En Revista Mexicana de Sociología, Año 48, Vol. 4, 1986, pp. 7-49.

Perló, M. y Ziccardi, A. "Desconcentrar o descentralizar: ésta es la cuestión". En La crisis en ciudades latinoamericanas. Martha Scheteingart (comp.), México, 1987.

Ziccardi, A. "La gobernabilidad de las metrópolis latinoamericanas". En Comercio Exterior, 10, Vol. 45, octubre, México, 1995. 